\title{
Study of Measurements for the Image Qualities of 3D TV
}

\author{
WU Dongyan, CAO Jiandong \\ Department of electronics and Communication Engineering \\ Suzhou Institute of Industrial Technology \\ Suzhou,CHINA \\ wudy@siit.cn, caojd@siit.cn
}

\begin{abstract}
This paper introduces the Polarization 3D technology and Active Shutter 3D technology, expounds the image quality index of $3 \mathrm{D}$ TV. This paper introduce the measurement of brightness/chroma/crosstalk/viewing angle. This paper propose center point measurement of 3D TV Eyes crosstalk testing method, enhances the picture catching ability, improve the precision and speed of testing crosstalk. This paper explain the viewing angle of $3 \mathrm{D}$ television, proposed one kind of measurement which can view test scheme, based on binocular crosstalk can determine the maximum angle of $3 D$ television.
\end{abstract}

\section{Keywords-3DTV; crosstalk; viewing angle}

\section{INTRODUCTION}

The rapid development of 3D technology, content production, stereo coding technology, stereo transmission and storage technology as well as the stereo display technology vigorous development, stereo TV industry has been a hot and bright, but the present stereo terminal market promotion effect is not ideal. First of all, the 3D content is still lacking, now 3D equipment is had already basically, but the lack of support for content, cannot be applied in large scale; secondly, stereo television image quality analysis, testing, no unified standard, resulting in $3 \mathrm{D}$ products to mass production, to promote the effect of general. The current 3D industry development, enterprise act of one's own free will, to develop their own technology and unique features, have quite one part consumer retains the $3 \mathrm{D}$ product, but unified stereo television image quality analysis, testing method and testing system has not been formed, the existing technology of 3D did not have a unified standard, unified problems become technology content problem after another restricted market development bottleneck.

Stereoscopic display technology is the technology to display the frontiers, which provides people with the three-dimensional sense of reproduction of the objective world and sense of reality, completely broke the original 2D display technology monopoly. With the continuous development of stereoscopic display technology, 3D TV has become the major 3D display terminal device. The 3D TV technology is a system engineering, which includes design, coding, transmission, etc. The image qualities of 3D TV decide the effect of 3D, so how to test the image qualities of 3D TV is an important research project. This paper mainly introduces the 3D television display technology and key technical indicators, elaborated with emphasis the binocular crosstalk and viewing angle characteristics of test scheme [1].

\section{DISPLAY TECHNOLOGY OF 3D TV}

Stereoscopic display is actually using the parallax principle; it makes two eyes respectively from different point of view to see the image, thereby reducing the stereo vision. Based on disparity realize stereo display technology, can be divided into glasses type $3 \mathrm{D}$ and naked 3D, the former must wear special 3D glasses, but the later no. This paper mainly discusses the display technology of glasses type 3D [2].

\section{A. Polarization 3D}

Polarizing $3 \mathrm{D}$ is the use of the principle of polarization to decompose the original image, the image is divided into first through a vertical polarized light and polarized light levels to two pictures, then 3D glasses respectively adopt different polarization direction of the polarized lenses, so that people around the eye can receive two pictures, and then after the synthesis of brain images, in the same screen display two pictures [3].

\section{B. Active Shutter $3 D$}

Active Shutter $3 \mathrm{D}$ is to a certain speed rotation transmission of left and right eye image, imaging end alternately displaying the right and the left eyes image. Viewers are required to wear a pair of LCD shutter glasses, consists of a sending end synchronous switch control, when the left eye image appears, the left liquid crystal light, right eye liquid crystal light; in contrast, when the right eye image, the right eye only liquid crystal light; the final two eyes can only see their own images.

\section{TESTING SIGNAL}

Because the present stereo TV show way for time division and beam mode and other modes, the test signal need to support multiple stereo signal format, can adapt to a wide range of test sample requirements. Stereoscopic test signal output formats are as follows.

\begin{tabular}{|l|l|}
\hline \multirow{2}{*}{ HDTV } & $72060 \mathrm{P} / 50 \mathrm{P} / 24 \mathrm{P}$ \\
\cline { 2 - 2 } & $108060 \mathrm{I} / 50 \mathrm{I} / 60 \mathrm{P} / 50 \mathrm{P} / 24 \mathrm{P}$ \\
\hline SDTV & $720 \times 576 \mathrm{I} 720 \times 576 \mathrm{P}$ \\
\hline \multirow{2}{*}{ PC } & $\begin{array}{l}800 \times 6001280 \times 10241024 \times 768 \\
1152 \times 8641280 \times 768\end{array}$ \\
\hline
\end{tabular}

\section{CHROMA}

The traditional chromaticity detection device in 2D television measurement is developed based on $3 \mathrm{D} \mathrm{TV}$, in the 
test, measurement points and the number of samples increased substantially. In the eyes of crosstalk testing method the proposed test scheme, need measurement screen multiple positions between the effects of different gray scale, measuring the number of times may reach hundreds of times. If using the traditional device test process more time. The project in the traditional chromaticity detection device based on $\mathrm{R} \& \mathrm{D}$, screen multipoint chromaticity detection device, to be used in full screen video as the basis, through the video analysis method for the measurement of every point on screen brightness, color coordinate information, enhance image capture capability, improve the crosstalk parametric test accuracy and speed.

The proposed test probe program are as follows: multi point testing device can be composed of a plurality of chromaticity detection device array, a plurality of detection points record screen color value. Each detection device to the point of less than 1 degrees, the transmission of data through a collection of multiple probe test, the method and single point repeatedly method has better consistency.

\section{CROSSTALK}

\section{A. Definition}

Ideally, the viewer's left eye sees only the left eye image, the right eye can only see the right eye image, and thus saw the ideal stereo image. However, the actual situation, in many viewing point to see is the left eye image crosstalk stereo image, crosstalk is the key factors of 3D TV. Because the crosstalk will lead to fusion and stereoscopic image distortion, which makes the viewers have different degrees of visual fatigue, thus achieving low crosstalk in high quality stereo display technology is very important. Image signal, a back light source, panel, shutter glasses and other factors caused the delay response will cause the eyes to characterization of crosstalk. In order to characterize the degree of crosstalk, we need to study the crosstalk of 3D TV.

\section{B. Testing scheme}

The stereoscopic display all of the reality of the brightness of the image containing an intermediate gray scale, the viewer will see the intermediate gray level crosstalk, and black / white signal and the response speed of intermediate gray scale signal response speed is different, so the black / white cross talk and intermediate gray scale crosstalk of different. Therefore, this paper uses the intermediate gray scale image crosstalk signal to test, the test signal input 5 level using gray $(0,25 \%, 50 \%, 75 \%$, $100 \%)[4]$.

Because the picture to the different locations of the crosstalk values are different, if the center of the frame crosstalk value is smaller, the image effect is good, and the surrounding location specific crosstalk value large enough, it is easy to produce visual fatigue. Through the research, we find that the stereo television image crosstalk value distribution does not rule, crosstalk research should consider three-dimensional image of the whole picture, at least to screen the multiple point as the measurement window.
Therefore, this paper adopts the whole picture test, the input signal on the screen in center point at the same time display window.

3D TV need work over an hour before testing; Measurement is as follows:

(1)3D glasses lens mounted on the left optical measuring device. Inputting level 0 from the start, with 25\% intervals, in turn increase the input signal level. Display measuring center points of the input level for the window $(0,25 \%, 50 \%$, $75 \%, 100 \%)$, in the reverse side center point input level $(0$, $25 \%, 50 \%, 75 \%, 100 \%)$ the window.

(2)We test the measurement point on the brightness of the 2 dimensional measurements, recording the input level measuring side and reverse side level combined eye portrait brightness measurement.

(3) The lateral window input level $\mathrm{M}$ is switched to a value, on all $\mathrm{M}$ input level repeated (2) operation.

(4) The measurement of lateral window input level $\mathrm{L}$ is switched to a value, on all 1 brightness level

(5) The glasses lens mounted on the right optical measuring device, for (3) (4) with the left eye and the right to repeat the same operation, measure and record data.

\section{BRIGHTNESS}

Brightness uniformity is one of the important indexes of brightness. In a stereoscopic television normal working conditions, we can measure brightness about 5 point of stereoscopic television screen, and calculates the brightness uniformity.

$$
P_{i}=\left|1-\frac{\left(L_{0}+L_{1}+L_{2}+L_{3}+L_{4}\right) / 5}{L_{i}}\right| \times 100 \% \text { (1) }
$$

$P_{i}$ is the brightness of different of the stereoscopic television.

\section{VIEWING ANGLE}

\section{A. Explanation}

When the viewers watch the $3 \mathrm{D} \mathrm{TV}$, viewers can obtain the effect of 3D maximum viewing angle, and watch the screen without distortion, fuzzy. The viewing angle of 3D TV includes vertical viewing angle and horizontal viewing angle. That is to say the 3D TV has a three-dimensional view angle, only in these angles; the observer's left (or right) eye saw the look of their left (or right) eye image. In addition to the regional, the observer will see either a left eye image or a right eye image aliasing, to do no parallax left eye image, namely the stereo view angle outside observer cannot see the right stereo image. Therefore, the viewing angle of $3 \mathrm{D} T V$ is the evaluation of $3 \mathrm{D} \mathrm{TV}$ performance and qualities [5].

\section{B. Testing scheme}

There is a way to the adjustment of different visual angle for testing binocular crosstalk. In order to test the convenience, can be used in a number of measurement points, can also use the central point measuring; can use 
multiple luminance signal, can also be directly used black $(0 \%)$ and white (100\%) signal simplified test.

There is a need to explain the test view angle, can keep the optical instrument for measuring the position unchanged, adjusting the measured 3D TV perspective; It also can maintain the 3D TV position unchanged, adjust the optical instrument for measuring angles.

Test for Horizontal View Angle of 3D TV:

3D TV center point must be the brightness measuring instrument in the same horizontal axis, and is working for more than an hour.

(1) The eye lens is mounted on the optical measuring device. The initial angle of horizontal is 0 degrees, and put in the measuring distance.

(2) In accordance with the binocular crosstalk measurement method, adding the initial measurement project input signal, whereby the input signal from the beginning of 0 , spaced at $25 \%$.The center point of the input level for the $\mathrm{L}$ window, in the reverse side input level for $\mathrm{M}$.

(3) Measuring and recording the results.

(4) To adjust the angle, repeat (2) (3).

(5) Completion of different visual angle eyes crosstalk measurement, which reflects the crosstalk based on binocular visual angle characteristics.

Test for Vertical View Angle of 3D TV:

The measurements of vertical view angle are similar to horizontal view angle. The only difference is that when adjusting the angle, vertical angle measurement is adjusted upward or downward adjustment, computational methods are the same.

\section{CONCLUSIONS}

(1) This paper analyzed the measurements for image of 3D TV, and put forward a set of test programs. Experimental results show that center point test can reflect the screen.

(2) This paper presents a 3D television viewing angle measurement method; the judgment should be based on the perspective of binocular crosstalk depending on the results of. In fact, view angle itself also has some subjective factors.

\section{ACKNOWLEDGMENT}

The authors thank the science and technology program of Suzhou (project number: SYG201248).

\section{REFERENCES}

[1] Kefeng Fan,Subing Zhang,Ling Cao.Study on the Standardization System of 3D TV :Information Technology \& Standardization Vol.9(2010),p.7

[2] Information on http://baike.baidu.com/view/2131807.htm

[3] Information on http://en.wikipedia.org/wiki/3D_television

[4] Dongyan Wu,Kefeng Fan,Shupo Bu,Zhan Zhao.Methods of Measurement for Eyes Crosstalk of 3D TV: Video Engineering Vol.36(6) (2012), p.87

[5] Information on http://it.sohu.com/20110128/n279138640.shtml 